\title{
Analysis of Patient Decision - Making Based on Satisfaction Effect
}

\author{
Wujun Cao, Lansheng Zhang, and Mengna Yan \\ Administrative Engineering College, Zhengzhou University, NO.100 Kexue Road, Zhengzhou, China \\ caowujun@zzu.edu.cn, q784612877@163.com
}

Keywords: cooperative medical treatment, patient treatment preferences; patient decisions factor; satisfaction utility model

\begin{abstract}
When patients make decisions to choose different levels of hospital, patients with different conditions vary in the will of decision-making factors. This paper presents a quantitative study of patient treatment preferences Satisfaction Utility Model (SUM). By surveying and statistic on patient preference for different treatment on the basis of decision-making factors, the paper introduced SUM to do the quantitative analysis, and finally got the levels of hospital satisfaction when patients with varying degrees in conditions choose different levels of hospitals, and this will provide some technical support for classification treatment of telemedicine services in cooperative medical treatment.
\end{abstract}

\section{Introduction}

In order to solve the problem of unfair allocation of medical resources effectively, and alleviate the people's livelihood of " To see a doctor is difficult and expensive", the Chinese government promulgated the "Opinions of the General Office of the State Council on Promoting the Construction of the Classification and Treatment System " on September 11, 2015. The aim is to achieve the target that the minor illness patient can into the primary hospital and the serious illness patient can into the center of the hospital, while with the supporting the upper and lower linkage mechanism, it can implement the basic policy that the central hospital help the basic hospital.

However, in our country, the status quo of the treat in the big hospital is prosperous, while the primary hospital is poor. Reasons for this status, in addition to the unreasonable distribution of medical resources, the patient's preference for choosing a hospital is also one of the most important factors. Because in the hospital medical service system, the patient not only involved in the entire service process, but also used to test the effectiveness of services, this special service relationship, making the patient's satisfaction with the study of treatment has a high practical significance. Therefore, in patients with treatment services, how to choose the best hospital based on the actual condition of the patient, is one of the important reasons for restricting Chinese medical reform.

Patient-treatment's Satisfaction Analysis (PSA) is a process that the patient analyzes the satisfaction of the hospital, with analyzing the time, cost and quality of treating. In response to health care reform, Michcel E. Porter proposed a new type of medical services to establish a comprehensive reform policy to maximize the interests of patients as a primary task (Michael E. Porter, Thomas H. Lee, 2013). Yanling Cai use this view applied to telemedicine, they attach great importance to emphasizing the value of patients involved in telemedicine, and establish a telemedicine system with the core of servicing patient (Yanling Cai, Yunkai Zhai, 2014). In response to the patient's decision-making factors, many scholars have studied the main factors when the treatment of patients with treatment decision-making. Among them, Jianjun Huang and other factors divide the affect of the patient treatment into six privacies, medical effects, medical staff attitude, the convenience of treatment process, medical expenses, red envelopes received, whether the medical staff respect the patient's indicators (Jianjun Huang, Yuhe Zeng, 2006). Huiqun Liao and other factors divide into five indicators of the satisfaction of Shenzhen outpatient patients, the registration fee, doctor's attitude, respected situation, pharmacy staff attitude, medical environment (Huiqun Liao, Xinyu Zeng, Yuqian Ren, Qiuxia Zeng, 2010). 
In addition, in the study of telemedicine, the country mainly focused on the study of regional health resources, the fairness and efficiency of the problem, as well as regional health information construction issues (Jieming Qu, Bo Tan, 2011). In foreign countries, in addition to the main study of the health of information resources, but also the main study of regional medical network site selection, size and capacity assessment of the problem (Park M H, Jo C, Bae E Y, 2012. Stiggelbout A M, Weijden T V D, Wit M P D, 2012). However, the current study of patient decision-making factors only stopped at the traditional medical research, no combination of telemedicine for research. These scholars in the analysis of patients to choose the basic factors of the hospital, almost no one is the hospital grade to sub-situation study, fewer scholars according to the size of the patient's disease to study the size of the situation.

This article uses the questionnaire survey to inferred the patient's decision-making factors and weights, with surveying the patient's choose treatment decision-making factors, in the two cases of the patients were in serious and not serious, and then on this basis, the application of satisfaction utility model was used to discuss the satisfaction value of the patient's treatment, and then to provide technical support for the Chinese level of diagnosis and treatment. Part 2 of the study researching the satisfaction model, part 3 to establish the satisfaction effect model, the fourth part analysis the patient treatment decision-making satisfaction, the fifth part discuss the value of this study, the research limitations and the research prospects in three directions.

\section{A Survey on Satisfaction Model}

Customer Satisfaction Index (CSI) is a kind of individual and subjective emotional reaction to the consumption object and consumption process after the customer's consumption, which is the quantitative index of the customer satisfaction level. Customer satisfaction has a certain deviation with the individual consumption, but overall, all customer satisfaction models are based on customer demand indicators. There are many ways to measure satisfaction at present, in this paper, we mainly study the habit preference of patients 'treatment, and use the satisfaction effect model to visualize the size of patients' satisfaction satisfactorily (Lijun Liang, Zixian Liu, Huaqiang Wang, 2013), and to compare and analyze the evaluation of patient's preference. It is of great significance to improve the medical environment and guide the medical reform.

\section{Satisfaction Utility Model}

Satisfaction Utility Model (SUM) is a decision-making method that uses the quantitative analysis of decision factors to establish an evaluation system and then to study the treatment of the entire patient.

\subsection{To establish a preliminary utility function}

The patient is an important decision maker for the selected hospital. When the satisfaction of the patient is investigated, the patient is the primary investigator of satisfaction, and his preference plays a decisive role in the overall medical service. Each patient has a different indicator of the choice of treatment options, and thus the utility value they receive not the same. In this paper, the patients choose the primary hospital or the central hospital as a reference index, the patient decision-making factors in the time, cost, quality of service as a secondary indicator to assess the establishment of a comprehensive utility system.

$$
u_{i k}=\beta_{i 1} x_{k 1}+\cdots+\beta_{i j} x_{k j}+\cdots+\beta_{i m} x_{k m}(1)
$$

Where $k=1,2,1$ represents the primary hospital, 2 represents the central hospital, $u_{i k}$ is the utility value of the $i$ th patient in the selected $k$-level hospital, and $\beta_{i j}$ is the preference coefficient when the $i$-th patient selecte $k$-level hospital for the $j$ factor; $x_{k j}$ is the level of the $j$ th decision-making factor that the patient refers to when choosing a k-level hospital. $\mathrm{m}$ represents the number of decision factors.

\subsection{Horizontal value not dimensioned}

The units of the decision factors in the equation (1) are different, so that the unit's dimensionless 
processing is required before the superposition is made, to make the units are unified. In order to deal with the different units to the operation of the trouble, all the decision factors into the level of $[-1,1]$ between the dimensionless data unified operation. Where the lowest level is -1 , the highest level is +1 , and the median is the mean value. And because the value of each decision-making factor has different measurement range, it is necessary to transform the weight value of the decision-making factor $\beta_{i j}$ to the relative preference coefficient $\sigma_{i j}$ at the same level.

$$
\sigma_{i j}=\frac{\beta_{i j}\left(X_{* j}-X_{j *}\right)}{\sum_{j=1}^{m}\left[\beta_{i j}\left(X_{* j}-X_{j *}\right)\right]}
$$

Where $\sigma_{\mathrm{ij}}$ is the relative preference coefficient of the i-th patient decision for the jth decision factors of the k-level hospital; $X_{* j}$ and $X_{* j}$ are the maximum and minimum values set for the $j$-th decision-making factor when selecting $k$-level hospitals.

\subsection{Determine The Final Utility Function}

The utility function of the level of decision factors obtained from equation (2) and relative preference coefficient is dimensionless:

$$
U_{i k}=\sigma_{i 1} X_{k 1}+\cdots+\sigma_{i j} X_{k j}+\cdots+\sigma_{i m} X_{k m}
$$

Where $U_{i k}$ is the utility value obtained for the $i$-th group of patients under investigation at the selected $k$-rank hospital; $X_{k j}$ is the dimensionless level of the $j$-th factor that is referenced when the $k$-level hospital is selected for the patient. When the patient chooses the primary hospital $(\mathrm{k}=1)$, the utility value $U_{i 1}$ obtained from the decision factors is greater than the utility value $U_{i 2}$ obtained from the decision-making factors when the central hospital $(\mathrm{k}=2)$ is selected. Choose $k$ grade hospital.

\subsection{Establish the overall satisfaction function}

First of all, according to the principle of patient satisfaction with the greater the better, this paper uses a large individual satisfaction function (4),

$$
D_{i}\left(\hat{X}_{i}\right)=\left\{\begin{array}{lc}
0 & \hat{X}_{i}<X_{i *} \\
\left(\frac{\hat{X}_{i}-X_{i *}}{T_{i}-X_{i *}}\right)^{s_{i}} \quad & X_{i *} \leq \hat{X}_{i} \leq T_{i} \\
1 & \hat{X}_{i}>T_{i}
\end{array}\right.
$$

function (3) relative to the utility value function, establishing the initial global function (5),

$$
D_{i k}\left(U_{i k}\right)=\left\{\begin{array}{l}
0 \quad U_{i k}<U_{i *} \\
\left(\frac{U_{i k}-U_{i k *}}{T_{i}-U_{i k}}\right)^{s_{i}} U_{i *} \leq U_{i k} \leq T_{i} \\
1 \quad U_{i k}>T_{i}
\end{array}\right.
$$

Where $D_{i k}$ is the satisfaction of the utility group obtained by the $k$-level hospital in the $i$-th group; $T_{i}$ are the lower bounds and target values of the $i$-th group of people who have access to the utility value of the $k$-level hospitals, and their values can be obtained from the data of the patient's decision-making questionnaire. The index $s_{i}$ represents the degree to which the utility value is satisfied and can be determined by the satisfaction of the different investigators on the utility value $U_{i k}$ close to the target utility value $T_{i}$.

Secondly, the weights of each decision-maker are given by selecting the weight of the decision-making factors $w_{i}$, and then all the utility values $D_{i k}$ are weighted geometrically averaged, as in the formula (6) , calculate the overall satisfaction function system:

$$
S_{k}\left(D_{1 k}\left(U_{1 k}\right), \cdots D_{i k}\left(U_{i k}\right), \cdots D_{n k}\left(U_{n k}\right)\right)=\left(\prod_{i=1}^{n} D_{i k}\left(U_{i k}\right)^{w_{i}}\right)^{1 / \sum w_{i}}
$$

Where $S_{k}$ is the total satisfaction of the utility value, $S_{k} \in[0,1] ; w_{i}$ is the weight of the $i$-th group of people participating in the decision-making, and they choose this level of hospital in the importance of these factors in their minds. 
Finally, the overall satisfaction function $S_{k}$ value of the size of the selected hospitals to sort, the larger the $S_{k}$ value on behalf of the choice of k-level hospital hospital more intense, the greater the satisfaction of the overall value.

$$
S=\max \left\{S_{1}, S_{2}, S_{3}, \cdots, S_{k}\right\}
$$

Table 1: A summary of the patient 's illness.

\begin{tabular}{c|c|c|c|c|c}
\hline \multirow{2}{*}{$\begin{array}{c}\text { Decision } \\
\text { situation }\end{array}$} & \multicolumn{2}{|c|}{ Serious illness } & \multicolumn{2}{c|}{ Not serious illness } & \multirow{2}{*}{ total } \\
\cline { 2 - 4 } & Percentage & $\begin{array}{c}\text { Total } \\
\text { proportion }\end{array}$ & Percentage & $\begin{array}{c}\text { Total } \\
\text { proportion }\end{array}$ & \\
\hline$\frac{\text { Choose to go to }}{\underline{\text { the hospital }}}$ & $\underline{\mathbf{8 1 . 3 2 \%}}$ & & $\underline{\mathbf{1 5 . 3 8 \%}}$ & & \\
$\begin{array}{c}\text { Central hospital } \\
\text { Primary hospital }\end{array}$ & $82.42 \%$ & $67.02 \%$ & $5.50 \%$ & $0.85 \%$ & $33.94 \%$ \\
$\frac{\text { Choose not to }}{\text { go to the }}$ & $17.58 \%$ & $14.30 \%$ & $94.50 \%$ & $14.53 \%$ & $14.42 \%$ \\
$\frac{\mathbf{1 8 . 6 8} \%}{\text { hospital }}$ & $18.68 \%$ & $\underline{\mathbf{8 4 . 6 2} \%}$ & $84.62 \%$ & $51.65 \%$ \\
Total & $\mathbf{1 0 0 . 0 0 \%}$ & $100.00 \%$ & $\mathbf{1 0 0 . 0 0 \%}$ & $100.00 \%$ & $100.00 \%$ \\
\hline
\end{tabular}

Table 2: Patient treatment based on the severity of the disease and the overall situation of the decision-making factors and the relative value of the value of the table.

\begin{tabular}{|c|c|c|c|c|c|c|}
\hline \multirow[b]{2}{*}{ Decision factors } & \multicolumn{2}{|c|}{ Overall situation } & \multicolumn{2}{|c|}{$\begin{array}{r}\text { Serious condition } \\
\text { Relativ }\end{array}$} & \multicolumn{2}{|c|}{$\begin{array}{r}\text { Not serious condition } \\
\text { Relativ }\end{array}$} \\
\hline & $\begin{array}{l}\text { Survey } \\
\text { value }\end{array}$ & $\begin{array}{l}\text { Relative } \\
\text { weight } \\
\text { value }\end{array}$ & $\begin{array}{c}\text { Survey } \\
\text { value }\end{array}$ & $\begin{array}{c}\text { e } \\
\text { weight } \\
\text { value }\end{array}$ & $\begin{array}{c}\text { Survey } \\
\text { value }\end{array}$ & $\begin{array}{c}\mathrm{e} \\
\text { weight } \\
\text { value }\end{array}$ \\
\hline High doctor level $X_{1}$ & 81 & 0.171 & 69 & 0.186 & 28 & 0.092 \\
\hline $\begin{array}{l}\text { Hospital is rich in } \\
\text { resources } X_{2}\end{array}$ & 63 & 0.133 & 61 & 0.164 & 16 & 0.053 \\
\hline Treatment fee $X_{3}$ & 68 & -0.143 & 51 & -0.138 & 51 & -0.167 \\
\hline $\begin{array}{l}\text { Hospital equipment } \\
\text { advanced } X_{4}\end{array}$ & 40 & 0.084 & 56 & 0.151 & 16 & 0.053 \\
\hline $\begin{array}{c}\text { Health insurance designated } \\
\text { hospitals } X_{5}\end{array}$ & 57 & 0.12 & 36 & 0.097 & 58 & 0.189 \\
\hline $\begin{array}{l}\text { Good reputation for } \\
\text { hospital } X_{6}\end{array}$ & 58 & 0.122 & 45 & 0.121 & 12 & 0.040 \\
\hline $\begin{array}{l}\text { Hospital service attitude is } \\
\qquad \operatorname{good} X_{7}\end{array}$ & 42 & 0.088 & 26 & 0.070 & 30 & 0.098 \\
\hline $\begin{array}{l}\text { Go to the hospital } \\
\text { convenient } X_{8}\end{array}$ & 26 & -0.055 & 20 & -0.054 & 64 & -0.209 \\
\hline $\begin{array}{l}\text { Treatment time is } \\
\text { reasonable } X_{9}\end{array}$ & 40 & -0.084 & 8 & -0.022 & 32 & -0.105 \\
\hline
\end{tabular}




\section{Analysis of patient satisfaction}

\subsection{Survey design}

This article examines the three directions of patient visits, respectively, the degree of the patient's condition, the hospital level of selecting, treatment decision-making factors, shown in Figure 1. The decision-making factors of patient treatment are divided into three first-level indicators, respectively, the cost of treatment, the time of treatment, the quality of treatment services; nine secondary factors, including time factors: hospital convenience, reasonable time; cost factors include: treatment costs, health insurance designated hospitals; quality of service factors include: a high level of doctors, hospital resources, hospital equipment, hospital reputation, hospital service attitude, summary data table in Table 2, decision factors shown in Figure 2.

\subsection{Questionnaire analysis}

According to the statistical analysis, the main decision-making factors in the patient's treatment, respectively, the degree of illness and the choice of hospital grade relationship between the table in Table 1, and patients in varying degrees of illness or in general, the choice of treatment of secondary with the values of the decision factors and the relative weights are shown in Table 2 .

When the patient's condition is different, the proportion of patients choose to visit the hospital is very different. When the patient was in serious condition, $81.32 \%$ of the patients chose to go to the hospital (82.42\% of the patients choose to go to the central hospital, only $17.58 \%$ of patients choose to go to primary hospitals), $18.68 \%$ of patients choose not to hospital;

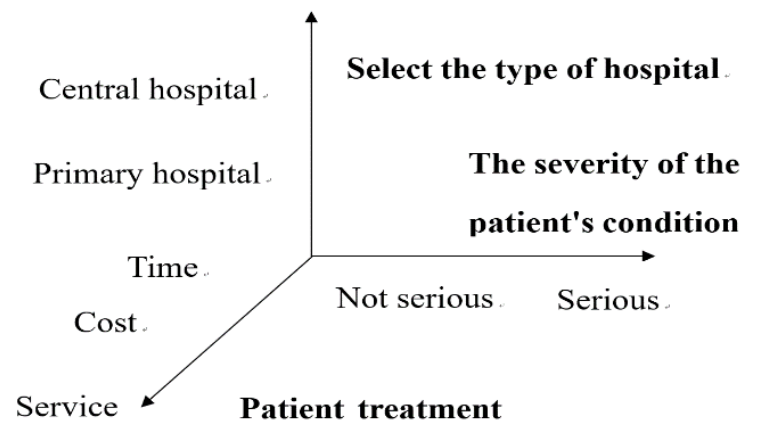

Figure 1: Patient visits to study ideas.

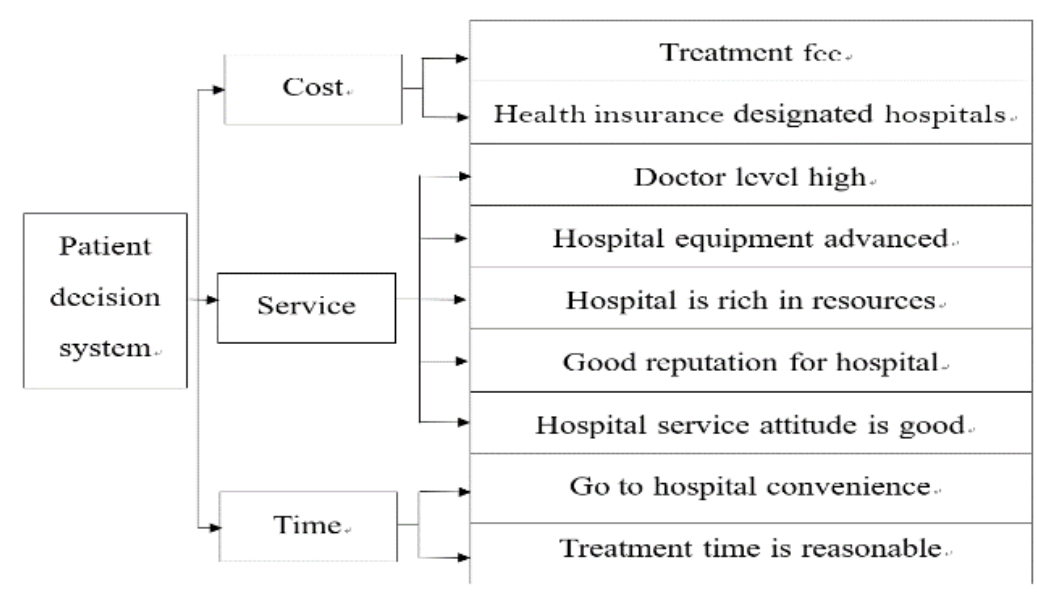

Figure 2: Summary of patient preference decision factors 
Table 3: The level of the primary decision-making factor selected by the patient to visit the hospital.

\begin{tabular}{|c|c|c|c|}
\hline Decision factors & \multicolumn{3}{|c|}{ Level value } \\
\hline High doctor level & Cure rate $>80 \%$ & Cure rate $>50 \%$ & Cure rate $<50 \%$ \\
\hline $\begin{array}{l}\text { Hospital is rich in } \\
\text { resources }\end{array}$ & rich & Generally rich & Not rich \\
\hline Treatment fee & $<100$ yuan & $<500$ yuan & $>500$ yuan \\
\hline $\begin{array}{l}\text { Hospital equipment } \\
\text { advanced }\end{array}$ & $\begin{array}{l}\text { Imported } \\
\text { equipment }\end{array}$ & $\begin{array}{l}\text { Domestic production of } \\
\text { advanced equipment }\end{array}$ & $\begin{array}{l}\text { Domestic } \\
\text { production for } \\
\text { ordinary equipment }\end{array}$ \\
\hline $\begin{array}{c}\text { Health insurance } \\
\text { designated hospitals }\end{array}$ & $\begin{array}{l}\text { Reimbursement of } \\
\text { more than } 50 \%\end{array}$ & $\begin{array}{l}\text { Reimbursement of } 50 \% \\
\text { or less }\end{array}$ & Not reimbursement \\
\hline $\begin{array}{l}\text { Good reputation for } \\
\text { hospital }\end{array}$ & Tertiary hospital & Secondary hospital & Primary hospital \\
\hline $\begin{array}{l}\text { Hospital service } \\
\text { attitude is good }\end{array}$ & Very good & Good & Not good \\
\hline $\begin{array}{c}\text { Go to the hospital } \\
\text { convenient }\end{array}$ & $<2 \mathrm{~km}$ & $<10 \mathrm{~km}$ & $>10 \mathrm{~km}$ \\
\hline $\begin{array}{l}\text { Treatment time is } \\
\text { reasonable }\end{array}$ & $<30$ min & $<60$ min & $>60 \mathrm{~min}$ \\
\hline
\end{tabular}

When the patient is not serious, only $15.38 \%$ of the total number of patients selected hospitals (almost all selected to the primary hospital), and $84.62 \%$ of patients choose not to go to the hospital.

When the patient in different degrees of disease and the overall situation in the choice of treatment programs, the degree of attention to the indicators significantly different. When the patient is in serious condition, the main focus on the doctor's technical level, the hospital's resources and other indicators; When the patient is not serious condition, the main focus is to go to the hospital's convenience and health insurance designated indicators and other indicators; And the overall consideration of the case, the patient focus on the doctor's technical level and medical expenses and other indicators. $\widehat{X}_{\imath}=X_{i} / \sum_{i=1}^{9} X_{i}$ is the result of the normalization of the index survey,

Which is the cost of treatment, to the convenience of the hospital, the rationality of the time of the three indicators of quantification and satisfaction values are negatively correlated, so the three indicators expressed as $-\widehat{X}_{l}$.

\subsection{Analysis of Patient Satisfaction}

\subsubsection{Horizontal value setting}

In order to be more persuasive to explain the level of these factors based on the reference to the Health Statistics Yearbook, as well as the doctor's technical level measurement indicators, treatment costs, medical reimbursement ratio, etc., the following table to determine the level of decision-making factors, table 3 summarizes the level of the primary decision-making factors that the patient chooses to visit the hospital, table 4 shows the level values in table 3 . table 5 shows the quantization values in table 4 using the equation (2), so that the data is in the range of $[-1,1]$. 
Table 4: Quantitative table of decision factors for patients.

\begin{tabular}{cccccc}
\hline Decision factors & Level value & $\begin{array}{c}\text { Central } \\
\text { hospital } \\
\text { level value }\end{array}$ & $\begin{array}{c}\text { Primary } \\
\text { hospital } \\
\text { level value }\end{array}$ \\
\hline $\begin{array}{c}\text { High doctor level } X_{1} \\
\text { Hospital is rich in resources } X_{2}\end{array}$ & 0.2 & 0.5 & 0.8 & 0.8 & 0.4 \\
Treatment fee $X_{3}$ & 100 & 500 & 1000 & 1000 & 600 \\
Hospital equipment advanced $X_{4}$ & 1 & 2 & 5 & 5 & 2 \\
Health insurance designated & 0.1 & 0.5 & 1 & 0.2 & 0.8 \\
hospitals $X_{5}$ & 1 & 3 & 5 & 5 & 3 \\
Good reputation for hospital $X_{6}$ & 1 & 3 & 5 & 4 & 3 \\
Hospital service attitude is & 1 & 3 & & 5 & 2000 \\
good $X_{7}$ & 2000 & 10000 & 30000 & 30000 & 30 \\
Go to the hospital convenient $X_{8}$ & 30 & 60 & 90 & 90 & \\
Treatment time is reasonable $X_{9}$ & 30
\end{tabular}

Table 5: The patient selects the dimensionless data sheet for the treatment program.

\begin{tabular}{cccccc}
\hline Decision factors & \multicolumn{2}{c}{ Level value } & $\begin{array}{c}\text { Central } \\
\text { Hospital } \\
\text { Score }\end{array}$ & $\begin{array}{c}\text { Primary } \\
\text { hospital } \\
\text { Score }\end{array}$ \\
\hline High doctor level $X_{1}$ & -1 & 0 & 1 & 1.00 & 0.00 \\
Hospital is rich in resources $X_{2}$ & -1 & 0 & 1 & 1.00 & 0.00 \\
Treatment fee $X_{3}$ & -1 & -1 & 1 & 1.00 & 0.11 \\
Hospital equipment advanced $X_{4}$ & -1 & 0 & 1 & 1.00 & -0.50 \\
Health insurance designated & -1 & -1 & 1 & -0.78 & 0.56 \\
hospitals $X_{5}$ & -1 & 0 & 1 & 1.00 & 0.00 \\
Good reputation for hospital $X_{6}$ & -1 & 0 & 1 & 0.50 & 0.00 \\
Hospital service attitude is good $X_{7}$ & -1 & -1 & 1 & 1.00 & -1.00 \\
Go to the hospital convenient $X_{8}$ & -1 & $/ 4$ & 1 & 1.00 & -1.00 \\
Treatment time is reasonable $X_{9}$ & -1 & 0 & 1 & 1.00 \\
\hline
\end{tabular}

According to the principle of patient's preferred value of treatment ---- the shorter the better of the patient's treatment, the lower the better of the cost, the higher the better of the diagnosis and treatment, and you can come to the ideal case of the target value: $X_{1}=1, X_{2}=1, X_{3}=$ $-1, X_{4}=1, X_{5}=1, X_{6}=1, X_{7}=1, X_{8}=-1, X_{9}=-1$

In the theory of microeconomics, utility is an important measure of a consumer's welfare, and consumers can bring satisfaction with each item in a unit of consumption. In the medical service, only when the utility value is greater than zero, the patient can accept, so the patient's minimum utility value is set to 0 , namely: $U_{1 *}=0, U_{2 *}=0$

\subsubsection{Patient treatment satisfaction utility value calculation}

(1) Analysis of Patient's Overall Situation Satisfaction

According to the intensity of the hospital grade selected in the case of the patient in the case of vague, calculate the statistical value $\beta_{i j}$ of the patient's overall preference and the relative weight coefficient $\sigma_{i j}$, and then replace the relative weight value $\sigma_{i j}$ into the formula (3), get the patient satisfaction overall satisfaction utility function,

$$
U_{k}=0.171 X_{1}+0.133 X_{2}-0.143 X_{3}+0.084 X_{4}+0.120 X_{5}+0.122 X_{6}+0.088 X_{7}-
$$
$0.055 X_{8}-0.084 X_{9}(8)$

Replace the level of the central hospital and the primary hospital level from table 5 into the overall satisfaction utility function of the patient's preference for treatment (8), we can calculate the 
patient's overall satisfaction utility value and target values to the central hospital and primary hospital: $U_{1}=0.1784, U_{2}=0.1485, T_{1}=1, T_{2}=1$

Assuming the index $s_{i}=1.2$, the patient's choice of treatment center hospitals and primary hospital satisfaction are: $D_{1}\left(U_{1}\right)=0.1600, D_{2}\left(U_{2}\right)=0.1230$

Because the reference object of the patient satisfaction is only the patient, the weight $w_{1}=$ $w_{2}=1$; then the patient overall satisfaction utility value is: $D_{1}=0.1600, D_{2}=0.1230$

As $D_{1}>D_{2}$, indicating that the patient in the overall situation, the central hospital value of the utility value is higher than the utility value of primary hospitals. It also shows that in the case of market information asymmetry, the patient feels more central hospital than the primary hospital. But the patient satisfaction target satisfaction 1, the central hospital and the overall satisfaction of the primary hospital from the target value of a greater gap, need to be improved.

(2) When the patient is in serious condition

First, based on the relative weight values of the patients who are established in Table 2, it is possible to obtain the decision function satisfaction function of the patients in the condition of serious condition

$U_{k 1}=0.186 X_{1}+0.164 X_{2}-0.138 X_{3}+0.151 X_{4}+0.097 X_{5}+0.121 X_{6}+0.070 X_{7}-0.054 X_{8}-$ $0.022 X_{9}(9)$

Then, according to the central hospital and the primary hospital decision-making factors relative to the level of value from table 5, combined with (9), we can calculate the patient selected central hospital utility value is $U_{1}=0.4113$ and the utility value of the hospital is $U_{2}=0.0396$,in patients with severe illness. The same as the above section to establish the standard value, you can calculate the central hospital satisfaction value and the satisfaction of the primary hospital value in the case of patient are in serious illness : $D_{11}=0.6503, D_{21}=0.0218$

It is clear that $D_{11}>D_{21}$, and the difference between the two, so that when the patient is in serious condition, patients choose the central hospital compared to the primary hospital will get greater satisfaction.

(3) When the patient is not serious

First, based on the relative weight values of the patients who are established in Table 8, it is possible to obtain the decision function satisfaction function of the patients in the condition of serious condition

$U_{k 2}=0.092 X_{1}+0.053 X_{2}-0.167 X_{3}+0.053 X_{4}+0.189 X_{5}+0.040 X_{6}+0.098 X_{7}-0.209 X_{8}-$ $0.105 X_{9}(10)$

Then, according to the central hospital and the primary hospital decision-making factors relative to the level of value from table 5,combined with (10), ,we can calculate the patient selected central hospital utility value is $U_{1}=-0.1314$ and the utility value of the hospital is $U_{2}=0.3750$,in patients with not severe illness. The same as the above section to establish the standard value, you can calculate the central hospital satisfaction value and the satisfaction of the primary hospital value in the case of patient are in serious illness : $D_{12}=0, D_{22}=0.5417$

Through the above calculation results, it can be clearly seen that when the patient is not serious, the patient's choice of central hospital satisfaction value is almost 0 , while the primary hospital satisfaction utility value of 0.5417 . This shows that the condition is not serious, choose to go to the central hospital patients are very dissatisfied with the choice of the nearby primary hospital is more economical.

But the patient's satisfaction target value of 1 , the minimum value of 0 , indicating that when the patient is not serious, the choice of central hospital treatment can make the patient is very dissatisfied, but the choice of primary hospital has a higher satisfaction utility value, but there is still a certain gap.

\section{Conclusions}

In this paper, combined with the satisfaction utility model, we can conclude that it is more 
reasonable for patients to chose central hospital when they are in serious illness, and then can chose primary hospital when they with serious illness, under the choice of hospital program decision-making with different degrees of disease. The study shows that this method needs to collect the relevant data of the relevant personnel more widely, so as to obtain a more accurate description, and then provide a more scientific basis for the patient to choose the hospital. In addition, it is necessary for the investigator to be more scientific and more sensible to make the choice of their own, in order to be more convincing. The same subject can be further studied on the basis of this method, taking into account many uncertainties (the uncertainty of patient treatment, the rationality of patient evaluation, the scientific nature of the survey population).

\section{References}

[1] Yanling Cai, Yunkai Zhai, 2014. Development of telemedicine system based on patients' value, Chinese Health Service Management. pp.644-646.

[2] Park M H, Jo C, Bae E Y, 2012. A comparison of preference of targeted therapy for metastatic renal cell carcinoma between the patient group and health care professional group in South Korea, Value in Health. 15(6), pp.933-939.

[3] Jianjun Huang, Yuhe Zeng, 2006. Logistic regression analysis of influencing factors of hospital choice,Chinese Journal of Hospital Statistics. pp.119-121.

[4] Lijun Liang, Zixian Liu, Huaqiang Wang, 2013. A Shared Decision-Making Model Based on DCE and Desirabilit y Function, Industrial Engineering and Management. 18(6), pp.79-82.

[5] Huiqun Liao, Xinyu, Zeng,Y uqian Ren, Qiuxia Zeng, 2010. Analysis on the satisfaction and influencing factors of outpatients in a hospital in Shenzhen city, Chinese Journal of Health Statistics. (04), pp.375-376.

[6] Stiggelbout A M, Weijden T V D, Wit M P D, 2012. Shared Decision making: really putting patients at the centre of healthcare, British Medical Journal. 344, pp. e245.

[7] Michael E. Porter, Thomas H. Lee, 2013. The strategy that will fix healthcare, Harvard Business Review. pp.2-19.

[8] Jieming Qu, Bo Tan, 2011. On the Reform of Medical Resources in Shanghai, Chinese Journal of Hospital Administration. 27(7), pp.499-501. 